\title{
The Influence of Literacy and Education on Online Health Information Seeking Behavior in Cancer Patients
}

\author{
Leziga T. Obiyo, ${ }^{* a}$ Chandy Ellimoottil, ${ }^{\mathrm{b}}$ William Adams, ${ }^{\mathrm{c}}$ Jessica H. Hannick ${ }^{\mathrm{b}}$, \\ Gopal N. Guptab \\ Loyola University Chicago Stritch School of Medicine, ${ }^{a}$ Loyola University Medical Center, \\ Department of Urology, ${ }^{b}$ and Public Health Sciences ${ }^{c}$, Loyola University Medical Center, Maywood \\ *lgbaanador@luc.edu
}

\begin{abstract}
:
Introduction: While the Internet has the potential to empower patients to make better informed medical decisions, little is known about $h$ ow patients search for health information. In this study, we characterize the online information seeking behavior of cancer patients and how it varies depending on literacy and education levels.

Methods: We performed a prospective study of patients with newly diagnosed urologic cancer who were asked to complete three tasks: 1) a demographic survey, 2) the Wide Range Achievement Test (WRAT-4) Blue Word Reading list, used as a proxy for literacy, and 3) a five minute self-directed Internet search about their cancer. We used monitoring software to examine the type (e.g. advertisements, cancer-specific, etc.) and duration of websites visited during the Internet search.

Results: Twenty-seven patients were enrolled. Among the seventeen patients who sought information from advertisement websites, $71 \%$ had below average WRAT-4 scores. Among the ten patients who sought information on non-advertisement websites, $80 \%$ had above average WRAT-4 scores. Of websites visited by those with only a high school education, $32.9 \%$ were advertisements. Alternatively, for those with at least a bachelor's degree, only $12.7 \%$ were advertisements.

Conclusion: We demonstrated that patients with low literacy and education achievement more frequently sought information from advertisement-oriented websites compared to those with higher literacy and education. These findings should encourage physicians to guide patients towards appropriate high quality websites, particularly patients with low literacy and/or education levels.
\end{abstract}

Keywords: computer literacy, information seeking behavior

\section{INTRODUCTION}

Of the 128 million Americans who have access to the Internet, $74 \%$ look for health information online. ${ }^{2}$ In the United States, 12.5 million health-related searches are conducted every day. ${ }^{3}$ Many people believe online health information educates, reassures and potentially empowers patients as they make critical health care decisions. In fact, $92 \%$ of patients believe the Internet enables them to make health decisions and improves their communication with their physician. ${ }^{3}$ Moreover, many physicians encourage patients to seek out information online as a method of promoting patientcentered care.

While it is clear patients use the Internet to seek health information, the methods they use to access websites and the type of websites they visit are largely unknown. It is plausible that patients searching the Internet have an inherent ability to delineate high quality websites from websites that are commercially biased or from untrusted sources. Patients may use their familiarity with trusted institutions or government-affiliated sites to guide their search. It is more likely, however, that patients lack the ability to differentiate poor from high quality websites. Patients may be distracted away from trusted websites and may instead be influenced by are advertisements. The latter scenario is likely more pronounced in populations of patients with lower literacy and education levels.

To better understand the landscape of online health information, previous investigators have used surveys, interviews, and website evaluation instruments (e.g, readability, cultural sensitivity, and quality). ${ }^{4-6}$ While these important measures can be used to reliably assess various features of health 
websites and elicit self-reported behavior, it is still unknown how patients behave when they are searching the Internet. In our study, we used website tracking software to address this gap in knowledge by monitoring the real-time behavior of patients as they seek information online. Our population includes patients of various literacy and education levels in order to identify differences in online health information seeking behavior. The findings of this study will help clinicians understand how patients search through online information and how literacy and education may alter this behavior. Ultimately, the findings in this study can be used by clinicians to guide their patients as they obtain online health information.

\section{MeTHODS}

\subsection{Study Population}

We designed a prospective survey study of patients with a new diagnosis of urologic cancer and an appointment with one of the urologic oncologists at Loyola University Medical Center Cardinal Bernadine Cancer Center. The study was approved by the Loyola University Institutional Review Board. Patients were identified by querying the urologic oncology clinic schedules for new patient appointments from July 2014 to March 2015. Patients identified as having been diagnosed with urologic cancer, but who had already undergone primary treatment were excluded. Potential enrollees were then contacted via telephone to ask if they were familiar with using the Internet. Those who reported that they were not familiar with the Internet were excluded.

\subsection{Tracking Information Seeking Behavior}

After patient consent was obtained at their clinic appointment, the patients were asked to complete several tasks. First, the patients completed a survey which included demographic information, highest education attainment (e.g. high school, some college, or bachelor's degree and beyond) and questions related to prior Internet usage (e.g. use at home, use to look up health information, and/or use to look up cancer-related information). Second, all patients completed the Wide Range Achievement Test: Fourth Edition (WRAT-4) Blue Word Reading list; used as a proxy for literacy. The WRAT-4 was selected for its ease of administration and standard acceptance as a tool for assessing literacy. The test was administered in two parts. Part 1 had 15 letters that the patient was asked to identify. Part 2 had 55 words that the patient was asked to read aloud. Poor literacy was defined as a standard WRAT-4 score that was below the standard mean of $100 .^{7}$ Following this, patients were directed to a computer and asked to spend five minutes on a self-directed Internet search about their cancer. The computer was equipped with software that tracked user activity. The software was selected for its ease of administration and non-interference with user activity. The monitoring software enabled monitoring and logging of all keystrokes, websites visits and online searches made by each study patient as they searched the Internet. After each patient completed their timed self-directed online search, the activity log was named and saved for future reference to ensure computer use correlated with the correct study patient. Study data were collected and managed using Research Electronic Data Capture.

(REDCap) tools hosted at Loyola University Health Sciences Division. REDCap is a secure, webbased application designed to support data capture for research studies, providing 1) an intuitive interface for validated data entry, 2) audit trails for tracking data manipulation and export procedures, 3) automated export procedures for seamless data downloads to common statistical packages, and 4) procedures for importing data from external sources ${ }^{8}$.

\subsection{Categorization of Websites}

We defined seven categories of websites. An advertisement was defined as a website that promoted the sale of a product, service, or therapy. A general health website was defined as a website that had information on a plethora of health issues (e.g. webmd.org). A general urology website was a domain that had information about the field of urology, urology practice, or a urology department at an academic medical institution or health system. A general cancer website was defined as a site that had information about cancer in general terms (e.g. cancer.gov). A cancer-specific website was defined as a website that had information about a particular cancer (e.g. kidneycancer.org). A cancer-specific treatment website was defined as a site that had information predominantly about treatment options for specific urologic cancer types. Websites that did not fit into the pre-selected categories, such as academic journals, were defined as other. 


\subsection{Statistical Analysis}

We first examined the type of websites visited by all patients. At the end of each patient encounter, survey results and tracking logs were uploaded into REDCap. Using the Blue Form subtest score forms, raw WRAT-4 scores from the word reading subtest were computed to generate the standard WRAT-4 score. We then stratified patients by WRAT-4 scores and highest education attainment to test the effect of literacy on the type of websites visited.

Scores from the WRAT-4 reading achievement test were adjusted for age and standardized $(M=$ $100, \mathrm{SD}=15$ ) using normative data provided by Psychological Assessment Resources, Inc. ${ }^{7}$ Normality was assessed using Q-Q plots, and extreme values were assessed using box plots. Nonparametric Wilcoxon-rank sum tests were used to make comparisons between standardized reading achievement score and type of website visited. Due to the

multitude of comparison analyses, significance values were adjusted for inflated Type I error using a conservative Bonferroni correction.

\section{RESULTS}

\subsection{Demographic Information}

A total of 42 patients were identified as study candidates over a nine-month period, of which 27 were enrolled. The remaining 15 patients were excluded either due to unfamiliarity with the Internet or desire not to participate. The enrolled patients represented diverse race and ethnic backgrounds and designated themselves as Asian (1, 3.7\%), Black or African American (6, 22.2\%), White (19, 70.4\%), and Unknown $(1,3.7 \%)$. Four patients $(14.8 \%)$ identified themselves as female and the remaining 23 $(85.2 \%)$ as male. The patients represented various education levels including those with only a high school diploma $(9,33.3 \%)$, some college education $(6,22.2 \%)$, and a bachelor's degree and beyond $(10,37 \%)$. Patient's urologic cancer diagnoses included prostate cancer $(15,55.6 \%)$ and kidney cancer $(12,44.5 \%)$.

\subsection{Overall Information Seeking Behavior}

According to survey results, 26 patients $(96.3 \%)$ reported previously using the Internet at home, 23 patients $(85.2 \%)$ used the Internet to look up health information, and 15 patients $(55.6 \%)$ used the Internet to find information about their cancer diagnosis. Based on the logs retrieved from the activity-monitoring software, study patients visited over 320 websites, of which 206 were unique and included for analysis. The most frequently visited website categories were general cancer $(n=63)$, general health $(n=55)$, and advertisements $(n=44)$ (Fig. 1).

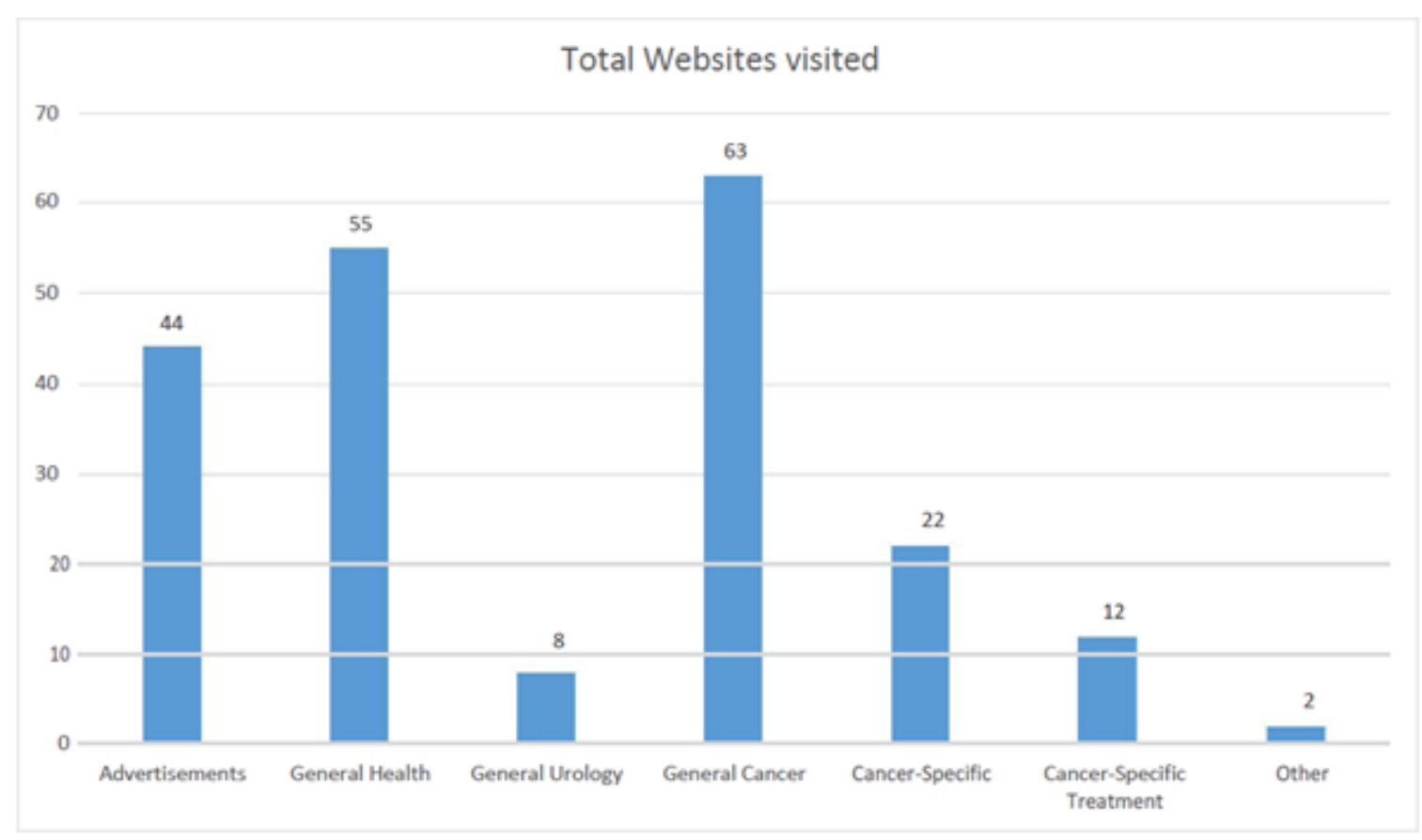

Figure1. General Cancer Websites were the most Frequent Website Category Visited by all Patients 


\subsection{Information Seeking Behavior of Patients by Literacy Levels}

Our patients demonstrated a mean standard WRAT-4 score of 98.81 with a standard deviation of 17.28. Five of the 27 patients had below average standard WRAT-4 scores $(<85)$. Of the 27 total recruited patients, $17(63.0 \%)$ visited advertisements and 10 patients (37.0\%) did not visit advertisements during their five-minute session. Of those 17 patients who visited advertisement websites, five had below average standard WRAT-4 scores and three patients had above average standard WRAT-4 scores ( $>115$ ) (Fig. 2a). Among the 10 patients who did not visit advertisements, all were within one standard deviation of the nationally normative population (i.e., none had a standard score below 85 or above 115). Interestingly, patients with an average standard WRAT-4 score or higher were less likely to seek health information about their diagnosis from cancer-specific treatment websites than patients with below average to average WRAT-4 scores (Fig. 2b). Most likely this finding was a chance observation due to our small sample size.



Figure2a. Histogram Demonstrates that Patients who Frequently Sought Health Information from Advertisements also had below Average WRAT-4 Standard Scores

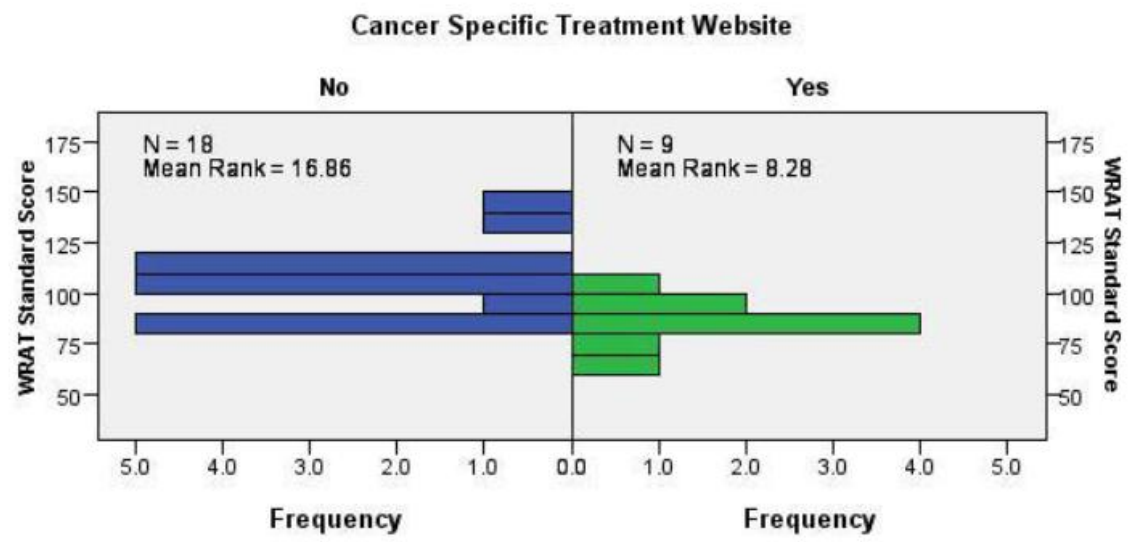

Figure2b. Histogram Demonstrates that Patients with at Least an Average WRAT-4 Standard Score are Less Likely to Seek Health Information from Cancer-Specific Treatment Websites

\subsection{Information Seeking Behavior of Patients by Education}

Of all websites visited by those with only a high school education, 32.9\% were advertisements compared to $12.7 \%$ by those with at least a bachelor's degree. Of all websites visited by patients with some college education, but not a bachelor's degree, 19.3\% were advertisements (Fig. 3).

Patients who collected information about their cancer diagnosis from advertisement-oriented websites had marginally lower reading ability after adjusting for age $(M d n=89, \mathrm{IQR}=84-103)$ compared to those who did not visit advertisements $(M d n=107, \mathrm{IQR}=104-111 ; p=.052)$. Interestingly, this was also true when examining cancer-specific treatment websites. Patients who directed their web browser towards a cancer-specific treatment website had significantly lower age-adjusted reading ability ( $M d n$ $=88, \mathrm{IQR}=80-90)$ when compared to patients who avoided such websites $(M d n=104, \mathrm{IQR}=89-$ $113 ; p=.01)$. There was no difference in reading ability between patients who collected information from general cancer, cancer-specific, general urology, general health, or other websites (all $p \geq .20$ ). 


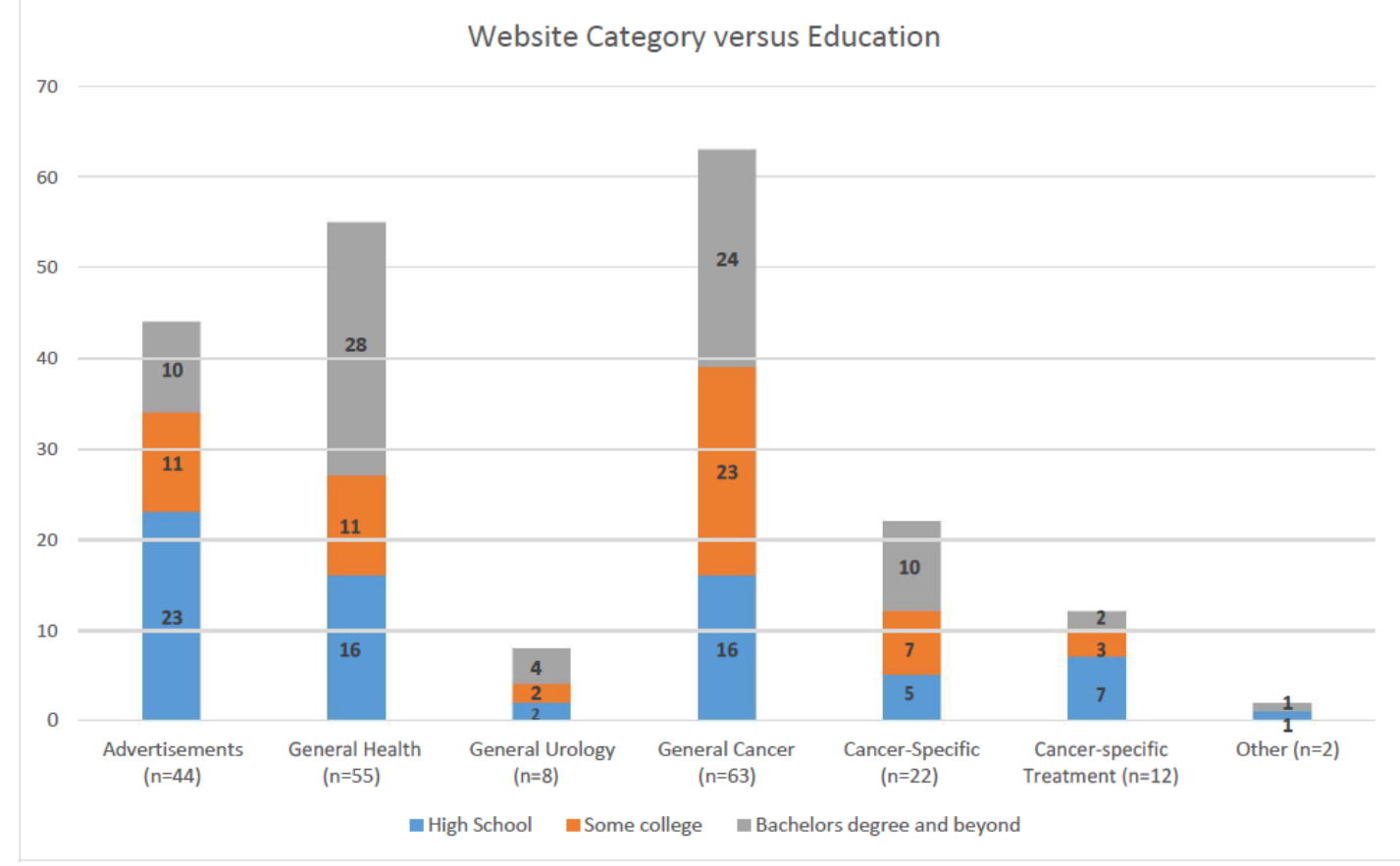

Figure3. Advertisements were a Large Proportion of the Websites Visited by Patients with only a High School Education, whereas, General Health and General Cancer Websites were the Highest Proportion of Websites Visited by Patients with a Bachelor's Degree and Beyond

\section{DisCUSSION}

In this study, we examined the information seeking behavior of newly diagnosed urologic cancer patients using website-tracking software. We found that most patients collected information from general cancer sites. It was encouraging to find that most patients gathered information from noncommercial websites that focused on cancer during their brief session online. When we stratified by literacy levels, however, we found that patients with lower literacy collected information from advertisements more frequently. Similarly, we found that patients with lower education attainment more frequently gathered information from advertisements. This may be due to a higher average readability of advertisement websites. Our findings demonstrated in Fig. 2b, however, suggest that patients with above average literacy were less likely to seek health information from cancer-specific treatment websites. These findings remained valid after adjusting for age. This finding was contrary to our hypothesis that patients with higher literacy seek information from more specific, higherquality websites. These findings may have swayed from initial expectations due to our study's small sample size. It was also possible that more literate and educated patients had already sought cancerspecific treatment information only prior to the study, thus biasing their study search results. Collectively, our findings suggest that patients with lower literacy and education attainment are more easily swayed by web advertisements.

Our findings are concerning because many Americans, including those with poor literacy turn to the Internet to learn about their disease, prognosis, and treatment options. The National Adult Literacy Survey (NALS) reported approximately 40 to 44 million American adults are functionally illiterate, and as many as 50 million American adults have marginal literacy skills. ${ }^{9}$

Patients can generally make a basic assessment of care received from the health care system. They have a difficult time, however, making a basic assessment of the quality of information available on the Internet as there is no general consensus about what constitutes high-quality health information. Previous research about the quality of websites states that advertisements are low quality because they are not traditionally considered credible sources. ${ }^{10,11}$ Our findings suggest that patients with low literacy and education levels are more likely to gather health information from poorer quality websites than their more literate and more educated counterparts.

Our study has several implications for clinicians and patients. Internet users may be overwhelmed by the generous amount of information available at the click of a mouse. Unlike journals 
publications which adhere to strict guidelines, the Internet allows the publication of information without subject to quality standards. Therefore, patients rely on limited medical knowledge to guide online searches about health conditions. ${ }^{4}$ Our findings support suggestions from previous work encouraging physicians to direct and support efforts to create quality assessment tools to identify websites with high-quality health information. ${ }^{12-14}$ It is imperative to disseminate this increased awareness about health literacy to create effective screening and

appropriately guide patients towards quality health information with the ultimate goal of improving patient's informed decision making. ${ }^{13,15,16}$

Our study has several limitations. First, our study has a small sample size which poses a challenge in interpreting results. Although studies with small sample sizes provide results quickly, they are subject to finding statistical relationships that might not otherwise exist in larger, more representative populations. Our study population was heavily male, however, this was inherent to the gender bias of urologic cancers towards males. Patients who reported prior Internet use for information about their cancer may have biased the searches they performed during the study. Additionally, the contrived nature of the study's short Internet search may have biased patient's actual search behavior compared to an untracked search in a private setting. Relating to the analysis of the patient searches, we could not be certain if advertisements were necessarily of less quality content than other sites, because a scale was not used to discriminate between high quality and low quality websites. Also, the content on the websites visited by the patients was not assessed to determine accuracy of the information presented. Finally, the dynamic nature of the Internet, may have altered the Internet search results that were generated for each study patient, thus inherently biasing potential information sources presented during their searches.

\section{Conclusion}

We demonstrated that patients with low literacy and education frequently sought information from advertisements compared to those with higher literacy and education when seeking information about genitourinary malignancies on the Internet. The utility of this study is to provide a methodology for researchers interested in studying patient education resources available on the Internet. While we chose urologic cancer as our disease of interest, the online health information seeking behaviors captured within this study would likely be applicable to all other diseases and treatments, including preventative services. The information gained from this study should enable researchers to be better informed about the websites that are used by patients.

The Internet has the potential to be a powerful resource to access health information. As patients shift to become a more active and commonly "Internet informed" consumer with the

widespread availability of the Internet; clinicians need to be mindful of the variability in literacy and education of their patient population and the importance of acknowledging this variability in order to guide these patients towards balanced and reputable online health information sources.

\section{REFERENCES}

[1] Friedman DB, Kao EK. A comprehensive assessment of the difficulty level and cultural sensitivity of online cancer prevention resources for older minority men. Prev Chronic Dis. 2008;5(1):A07.

[2] Fox S. The Social Life of Health Information. 2009. http://www.webcitation.org/5zmDS2IBq.

[3] Eysenbach G. The Impact of the Internet on cancer outcomes. CA Cancer J Clin. 2003; 53(6):356-371.

[4] Ilic D, Risbridger G, Green S. Searching the internet for information on prostate cancer screening: An assessment of quality. Urology. 2004;64(1):112-116. doi:10.1016/j.urology.2004.02.027.

[5] Ellimoottil C, Polcari A, Kadlec A, Gupta G. Readability of websites containing information about prostate cancer treatment options. J Urol. 2012; 188(6):2171-2175. Doi:10.1016/ j.juro.2012.07.105.

[6] Berland, GK, Elliott, MN, Morales, LS, Algazy, JI, Kravitz, RL, Broder, MS E al. Health Information on the Internet: accessibility, quality, and readability in English and Spanish. JAMA. $2001 ; 285(20): 2612-2621$. 
[7] Wilkinson GS, Robertson GJ. Wide Range Achievement Test: Professional Manual. 4th Editio. PAR, Inc.; 2006.

[8] Harris P a., Taylor R, Thielke R, Payne J, Gonzalez N, Conde JG. Research electronic data capture (REDCap)-A metadata-driven methodology and workflow process for providing translational research informatics support. $J$ Biomed Inform. 2009; 42(2):377-381. doi:10.1016/j.jbi.2008.08.010.

[9] Kirsch IS, Jungeblut A, Jenkins L, Kolstad A. Adult literacy in America: A first look at the findings of the National Adult Literary Survey. 1993. https://www-ncjrs-gov.proxy.lib.pdx.edu/ App/abstractdb/AbstractDBDetails.aspx ?id=144980.

[10] Eysenbach G, Diepgen TL. Information in practice information. Bmj. 1998; 317(November):1496-1502.

[11] Cline RJ, Haynes KM. Consumer health information seeking on the Internet: the state of the art. Health Educ Res. 2001; 16(6):671-692. doi:10.1093/her/16.6.671.

[12] Dubowicz A, Schulz PJ. Medical Information on the Internet: A Tool for Measuring Consumer Perception of Quality Aspects. Eysenbach G, ed. Interact J Med Res. 2015; 4(1):e8. doi:10.2196/ijmr.3144.

[13] Pautler SE, Tan JK, Dugas GR, et al. Use of the internet for self-education by patients with prostate cancer. Urology. 2001; 57(2):230-233. doi:S0090-4295(00)01012-8 [pii] ET $2001 / 02 / 22$.

[14] Lawrentschuk N, Abouassaly R, Hackett N, Groll R, Fleshner NE. Health Information Quality on the Internet in Urological Oncology: A Multilingual Longitudinal Evaluation. Urology. 2009; 74(5):1058-1063. doi:10.1016/j.urology.2009.05.091.

[15] Listed N authors. Health literacy: Report of the Council on Scientific Affairs. JAMA. 1999; 281(6):552-557.

[16] Hansberry DR, Agarwal N, Baker SR. Health Literacy and Online Educational Resources: An Opportunity to Educate Patients. Am J Roentgenol. 2015; 204(1):111-116. doi:10.2214/AJR.14.13086. 